PROCEEDINGS OF THE

AMERICAN MATHEMATICAL SOCIETY

Volume 136, Number 6, June 2008, Pages 2235-2244

S 0002-9939(08)09039-4

Article electronically published on February 11, 2008

\title{
ASYMPTOTICS OF MATRIX INTEGRALS AND TENSOR INVARIANTS OF COMPACT LIE GROUPS
}

\author{
MICHAEL STOLZ AND TATSUYA TATE
}

(Communicated by Mikhail Shubin)

\begin{abstract}
In this paper we give an asymptotic formula for a matrix integral which plays a crucial role in the approach of Diaconis et al. to random matrix eigenvalues. The choice of parameter for the asymptotic analysis is motivated by an invariant-theoretic interpretation of this type of integral. For arbitrary regular irreducible representations of arbitrary connected semisimple compact Lie groups, we obtain an asymptotic formula for the trace of permutation operators on the space of tensor invariants, thus extending a result of Biane on the dimension of these spaces.
\end{abstract}

\section{INTRODUCTION}

Let $G$ be a compact connected Lie group, and let $\left(V_{\lambda}, \rho_{\lambda}\right)$ be an irreducible representation of $G$ with highest weight $\lambda$. Consider the matrix integral

(1)

$$
\int_{G}\left(\operatorname{Tr} \rho_{\lambda}(g)\right)^{a_{1}}\left(\operatorname{Tr} \rho_{\lambda}\left(g^{2}\right)\right)^{a_{2}} \ldots\left(\operatorname{Tr} \rho_{\lambda}\left(g^{r}\right)\right)^{a_{r}}\left(\overline{\operatorname{Tr} \rho_{\lambda}(g)}\right)^{b_{1}} \ldots\left(\overline{\operatorname{Tr} \rho_{\lambda}\left(g^{r}\right)}\right)^{b_{r}} d \omega_{G}(g),
$$

where $\left\{a_{j}\right\}_{j=1}^{r},\left\{b_{j}\right\}_{j=1}^{r}$ are fixed sequences of nonnegative integers and $\omega_{G}$ denotes normalized Haar measure on $G$. In the case that $\left(V_{\lambda}, \rho_{\lambda}\right)$ is the standard representation of the unitary group $G=\mathrm{U}_{n}$, the integral (1) is nothing else than the $\left(a_{1}, \ldots, a_{r}, b_{1}, \ldots, b_{r}\right)$-moment of the random vector

$$
\left(\operatorname{Tr}(g), \ldots, \operatorname{Tr}\left(g^{r}\right), \overline{\operatorname{Tr}(g)}, \ldots, \overline{\operatorname{Tr}\left(g^{r}\right)}\right),
$$

where $g$ is chosen from $\mathrm{U}_{n}$ according to the Haar measure. It has been proven by Diaconis and Shahshahani in DS (see also DE) that for $n$ large enough, (1) coincides with the $\left(a_{1}, \ldots, a_{r}, b_{1}, \ldots, b_{r}\right)$-moment of a vector of independent complex Gaussian random variables of suitable variances, and consequently, the vector (2) converges in distribution to this Gaussian vector as the matrix size $n$ tends to infinity. It has been observed $([\mathrm{BR}],[\mathrm{St}])$ that the Diaconis-Shahshahani result is based on the fact that the integral (1) can be expressed as

$(3)$

$$
\int_{G} \prod_{j=1}^{r} \operatorname{Tr}\left(\rho_{\lambda}(g)^{j}\right)^{a_{j}} \operatorname{Tr}\left(\rho_{\lambda}^{*}(g)^{j}\right)^{b_{j}} d \omega_{G}(g)=\operatorname{Tr}\left(\left.\left(\sigma_{k_{a}} \otimes \sigma_{k_{b}}\right)(s, t)\right|_{\left[V_{\lambda}^{\otimes k_{a}} \otimes\left(V_{\lambda}^{*}\right)^{\otimes k_{b}}\right]^{G}}\right) .
$$

Received by the editors October 19, 2006, and, in revised form, December 12, 2006.

2000 Mathematics Subject Classification. Primary 22E46; Secondary 43A99.

Key words and phrases. Asymptotic analysis, compact Lie groups, invariant theory, matrix integrals.

(c)2008 American Mathematical Society Reverts to public domain 28 years from publication 
Here we write $k_{a}=\sum_{j=1}^{r} j a_{j}$ and define $k_{b}$ analogously. $\sigma_{k_{a}}$ denotes the obvious representation of the symmetric group $\mathfrak{S}_{k_{a}}$ on $V_{\lambda}^{\otimes k_{a}}$, and $\sigma_{k_{b}}$ is its analogue on $\left(V_{\lambda}^{*}\right)^{\otimes k_{b}}$, where the representation $\left(V_{\lambda}^{*}, \rho_{\lambda}^{*}\right)$ is contragredient to $\left(V_{\lambda}, \rho_{\lambda}\right) . s \in \mathfrak{S}_{k_{a}}$ has cycle type $\left(1^{a_{1}} \ldots r^{a_{r}}\right)$ and $t \in \mathfrak{S}_{k_{b}}$ has cycle type $\left(1^{b_{1}} \ldots r^{b_{r}}\right)$.

$$
\begin{aligned}
& {\left[V_{\lambda}^{\otimes k_{a}} \otimes\left(V_{\lambda}^{*}\right)^{\otimes k_{b}}\right]^{G}} \\
& \quad=\left\{T \in V_{\lambda}^{\otimes k_{a}} \otimes\left(V_{\lambda}^{*}\right)^{\otimes k_{b}}:\left(\rho_{\lambda}^{\otimes k_{a}} \otimes\left(\rho_{\lambda}^{*}\right)^{\otimes k_{b}}\right)(g) T=T \text { for all } g \in G\right\}
\end{aligned}
$$

is the space of invariants of the $G$-action $\rho_{\lambda}^{\otimes k_{a}} \otimes\left(\rho_{\lambda}^{*}\right)^{\otimes k_{b}}$. Diaconis and Shahshahani study the integral (11) for a sequence $\left(G_{n}\right)$ of classical groups of increasing rank, fixing the parameters $a=\left(a_{1}, \ldots, a_{r}\right)$ and $b=\left(b_{1}, \ldots, b_{r}\right)$. Since they consider standard representations of classical groups, substantial information about the right-hand side of (3) has become available since the epoch-making work of Weyl $(\overline{\mathrm{Wy}}])$ and can be used to evaluate the left-hand side.

In the present paper, we depart from the Diaconis-Shahshahani framework in two ways. Firstly, we consider arbitrary regular irreducible representations of arbitrary compact connected semisimple Lie groups. In this generality, one has a priori only poor control of the right-hand side of (3). So we will directly attack the left-hand side of this equation and thus obtain some asymptotic information about the spaces of invariants on the right-hand side. Secondly, we fix a group $G$ and a representation $V_{\lambda}$ and let the parameters $k_{a}, k_{b}$ of the tensor powers (and hence the moment parameters $a, b$ ) tend to infinity. This may be thought of as a thermodynamic limit of a particle system, rather than a random matrix limit. For our asymptotic analysis we will use techniques which were developed by Biane ([B] $)$, Klyachko and Kurtaran $([\mathrm{KK}])$, and Tate and Zelditch ([TZ]). Our results should be compared to those of Biane $([\mathrm{B}]$ ) and Kuperberg $([\mathrm{K}])$. An asymptotic result of a different kind for growing tensor powers of a fixed representation has recently been obtained by Collins and Śniady in [CS, Thm. 17].

Here is the setup for our main theorems: Assume that the compact connected Lie group $G$ is semisimple, and that the highest weight $\lambda$ of the fixed irreducible representation $\left(V_{\lambda}, \rho_{\lambda}\right)$ is regular, i.e., is in the interior of a Weyl chamber. Fix a maximal torus $T$ in $G$. Denote by $W$ the Weyl group. Write $\mathfrak{t}$ for the Lie algebra of $T$, and $\mathfrak{t}^{*}$ for its dual space. $I:=$ ker exp $\subset \mathfrak{t}$ is the integral lattice, and its dual $I^{*}:=\left\{\varphi \in \mathfrak{t}^{*}: \varphi(I) \subseteq \mathbb{Z}\right\}$ is the weight lattice. Let $\Lambda^{*} \subset \mathfrak{t}^{*}$ be the abelian group generated by the roots, i.e. the root lattice, and write $\Lambda:=\left(\Lambda^{*}\right)^{*}$ for its dual. It is well known that $\Lambda^{*} \subseteq I^{*}$, hence $I \subseteq \Lambda$, and that the group $\Pi(G):=\Lambda / I$ is a finite abelian group. It can be regarded as a subgroup of $T \cong \mathfrak{t} / I$. Write $\pi$ for the canonical projection of $T$ onto $T / \Pi(G)$. For any $\mu \in I^{*}$ write $\mathrm{m}_{\lambda}(\mu)$ for its multiplicity in $V_{\lambda}$. Then, the set of all weights of $V_{\lambda}$ is $M_{\lambda}:=\left\{\mu \in I^{*}: \mathrm{m}_{\lambda}(\mu) \neq 0\right\}$.

Fix a smooth function $f>0$ on $G$ and sequences $\alpha=\left(\alpha_{1}, \ldots, \alpha_{r}\right)$ and $\beta=$ $\left(\beta_{1}, \ldots, \beta_{r}\right)$ of nonnegative integers. For a positive integer $N$ set $a_{j}:=a_{j}(N):=$ $N \alpha_{j}, b_{j}:=N \beta_{j}(j=1, \ldots, r)$ and write $a=\left(a_{1}, \ldots, a_{r}\right), b=\left(b_{1}, \ldots, b_{r}\right)$. Furthermore, we set

$$
|\alpha|:=\sum_{j=1}^{r} \alpha_{j}, \quad k_{\alpha}:=\sum_{j=1}^{r} j \alpha_{j}, \quad l_{\alpha}:=\sum_{j=1}^{r} j^{2} \alpha_{j}
$$


and define $|\beta|, k_{\beta}, l_{\beta}, k_{a}$, etc. analogously. Throughout the paper we will assume that $k_{\alpha}=k_{\beta}$. We will study the integrals

$$
\begin{gathered}
I_{N}=I_{N}(f, \alpha):=\int_{G} \prod_{j=1}^{r}\left(\operatorname{Tr}\left(\rho_{\lambda}\left(g^{j}\right)\right)\right)^{N \alpha_{j}} f(g) d \omega_{G}(g) \text { and } \\
K_{N}=K_{N}(f, \alpha, \beta):=\int_{G} \prod_{j=1}^{r}\left(\operatorname{Tr}\left(\rho_{\lambda}\left(g^{j}\right)\right)\right)^{N \alpha_{j}}{\overline{\left(\operatorname{Tr}\left(\rho_{\lambda}\left(g^{j}\right)\right)\right)}}^{N \beta_{j}} f(g) d \omega_{G}(g) .
\end{gathered}
$$

Writing $\Phi_{+}$for the set of positive roots of $(G, T)$, we define a polynomial $\kappa$ on $\mathfrak{t}$ by

$$
\kappa(x)=\prod_{\alpha \in \Phi_{+}}\langle\alpha, x\rangle .
$$

Finally, we define $A_{\lambda} \in \operatorname{Hom}_{\mathbb{C}}\left(\mathfrak{t}, \mathfrak{t}^{*}\right) \cong \mathfrak{t}^{*} \otimes \mathfrak{t}^{*}$ by

$$
A_{\lambda}=\frac{1}{\operatorname{dim} V_{\lambda}} \sum_{\mu \in M_{\lambda}} m_{\lambda}(\mu) \mu \otimes \mu \text {. }
$$

Since $G$ is assumed to be semisimple, and $\lambda$ to be regular, $A_{\lambda}$ is known to be positive definite (see [TZ]).

Now we are in a position to state our main results, to be proven in Sections 3 5 below.

Theorem 1. Assume that $\operatorname{gcd}\left\{j: \alpha_{j} \neq 0\right\}=1$. Then

$$
I_{N}(f, \alpha)=\frac{(2 \pi)^{d}\left(\operatorname{dim} V_{\lambda}\right)^{N|\alpha|} \kappa\left(A_{\lambda}^{-1} \rho\right)}{\left(2 \pi l_{\alpha} N\right)^{(\operatorname{dim} G) / 2} \sqrt{\operatorname{det} A_{\lambda}}}\left(\sum_{h \in \Pi(G)} \nu_{N k_{\alpha} \lambda}(h) f^{G}(h)+O\left(N^{-1 / 2}\right)\right)
$$

where $d$ is the number of positive roots, $\rho$ is half the sum of the positive roots, $f^{G}$ is the class function $f^{G}(g)=\int f\left(x^{-1} g x\right) d \omega_{G}(x)$, and $\nu_{N k_{\alpha} \lambda}$ is the character on $\Pi(G)$ determined by the weight $N k_{\alpha} \lambda$ :

$$
\nu_{N k_{\alpha} \lambda}(h)=e^{2 \pi \sqrt{-1} N k_{\alpha}\left\langle\lambda, \psi_{h}\right\rangle}
$$

$\psi_{h} \in \Lambda$ being a coset representative of $h \in \Lambda / I=\Pi(G)$.

Theorem 2. Suppose that $\operatorname{gcd}\left\{j: \alpha_{j} \neq 0\right.$ or $\left.\beta_{j} \neq 0\right\}=1$. Then

$$
K_{N}(f, \alpha, \beta)=\frac{(2 \pi)^{d}\left(\operatorname{dim} V_{\lambda}\right)^{N(|\alpha|+|\beta|)} \kappa\left(A_{\lambda}^{-1} \rho\right)}{\left(2 \pi N\left(l_{\alpha}+l_{\beta}\right)\right)^{(\operatorname{dim} G) / 2} \sqrt{\operatorname{det} A_{\lambda}}}\left(\sum_{h \in \Pi(G)} f^{G}(h)+O\left(N^{-1 / 2}\right)\right) .
$$

Remark 1.1. Note that in Theorem [1] the leading term vanishes if $N k_{\alpha} \lambda=k_{a} \lambda$ is not contained in the lattice $\Lambda^{*}$ and if $f \equiv 1$. In fact, in this case one has $\left[V_{\lambda}^{\otimes k_{a}}\right]^{G}=\{0\}$; hence $I_{N}(1, \alpha)=0$ by (3). This is because the existence of a nontrivial invariant implies that there is a sequence $\mu_{1}, \ldots, \mu_{k_{a}}$ of weights for the irreducible representation $V_{\lambda}$ such that $\mu_{1}+\cdots+\mu_{k_{a}}=0$. Recall the well-known fact (see [TZ]) that, if the highest weight $\lambda$ is regular, then the root lattice $\Lambda^{*}$ is spanned by the differences $\mu-\mu^{\prime}$ between two weights $\mu, \mu^{\prime}$ for $V_{\lambda}$. This implies that

$$
1=e^{2 \pi \sqrt{-1}\left\langle\mu_{1}+\cdots+\mu_{k_{a}}, \psi\right\rangle}=e^{2 \pi \sqrt{-1} k_{a}\langle\lambda, \psi\rangle}
$$

for any $\psi$ in the dual $\Lambda$ of the root lattice $\Lambda^{*}$, and hence $k_{a} \lambda \in \Lambda^{*}$. 
Remark 1.2. In view of (3), Theorems 1 and 2 give asymptotic formulae for the trace of permutations on the space of tensor invariants. Specializing to the identity permutation, one obtains the asymptotics of the dimension of these spaces. Specifically, taking in Theorem $1 \alpha_{j}=0$ for $j \geq 2$ and $\alpha_{1}=1, f \equiv 1$, and assuming that $\lambda$ is in the root lattice $\Lambda^{*}$, we obtain an asymptotic formula for the dimension of the space of tensor invariants, namely

$$
\operatorname{dim}\left[V_{\lambda}^{\otimes N}\right]^{G}=\frac{|\Pi(G)|\left(\operatorname{dim} V_{\lambda}\right)^{N} \kappa\left(A_{\lambda}^{-1} \rho\right)}{(2 \pi)^{(\mathrm{rk} G) / 2} N^{(\operatorname{dim} G) / 2} \sqrt{\operatorname{det} A_{\lambda}}}\left(1+O\left(N^{-1 / 2}\right)\right) .
$$

This formula has been obtained by Biane in [B] (see also [TZ]).

\section{Matrix INTEGRALS AND TENSOR INVARIANtS}

Before turning to the proof of Theorems 11 and 2 we provide a short and selfcontained proof of equation (3), on which the invariant-theoretic interpretation of the integrals $I_{N}$ and $K_{N}$ is based. Write $H:=\left[V_{\lambda}^{\otimes k_{a}} \otimes\left(V_{\lambda}^{*}\right)^{\otimes k_{b}}\right]^{G}$. Then, the orthogonal projection onto $H$ is given by

$$
\pi_{H}(T)=\int_{G}\left(\rho_{\lambda}^{\otimes k_{a}} \otimes\left(\rho_{\lambda}^{*}\right)^{\otimes k_{b}}\right)(g) T d \omega_{G}(g), \quad T \in V_{\lambda}^{\otimes k_{a}} \otimes\left(V_{\lambda}^{*}\right)^{\otimes k_{b}} .
$$

For $A \in \operatorname{End}_{G}\left(V_{\lambda}^{\otimes k_{a}} \otimes\left(V_{\lambda}^{*}\right)^{\otimes k_{b}}\right)$, then,

$$
\operatorname{Tr}\left(\left.A\right|_{H}\right)=\operatorname{Tr}\left(A \pi_{H}\right)=\operatorname{Tr}\left(\pi_{H} A\right) .
$$

We thus obtain

Lemma 2.1. For any $A \in \operatorname{End}_{G}\left(V_{\lambda}^{\otimes k_{a}} \otimes\left(V_{\lambda}^{*}\right)^{\otimes k_{b}}\right)$, we have

$$
\begin{aligned}
\operatorname{Tr}\left(\left.A\right|_{H}\right) & =\int_{G} \operatorname{Tr}\left(\left(\rho_{\lambda}^{\otimes k_{a}} \otimes\left(\rho_{\lambda}^{*}\right)^{\otimes k_{b}}\right)(g) A\right) d \omega_{G}(g) \\
& =\int_{G} \operatorname{Tr}\left(A\left(\rho_{\lambda}^{\otimes k_{a}} \otimes\left(\rho_{\lambda}^{*}\right)^{\otimes k_{b}}\right)(g)\right) d \omega_{G}(g) .
\end{aligned}
$$

In view of this, (3) is implied by the following well-known lemma (see [DS, Ra]):

Lemma 2.2. Let $V$ be a d-dimensional complex vector space, $B \in \operatorname{End}_{\mathbb{C}}(V)$, and $s \in \mathfrak{S}_{k}$ a permutation of type $\left(1^{a_{1}} 2^{a_{2}} \ldots r^{a_{r}}\right)$; hence $k=k_{a}=\sum_{j=1}^{r} j a_{j}$. Let $\mathfrak{S}_{k}$ act on $V^{\otimes k}$ via $\sigma_{k}(s)\left(\bigotimes_{i=1}^{k} v_{i}\right):=\bigotimes_{i=1}^{k} v_{i s^{-1}}$. Then the trace of $B^{\otimes k} \sigma_{k}(s) \in$ $\operatorname{End}_{\mathbb{C}}\left(V^{\otimes k}\right)$ is

$$
\operatorname{Tr}\left(B^{\otimes k} \sigma_{k}(s)\right)=\prod_{j=1}^{r} \operatorname{Tr}\left(B^{j}\right)^{a_{j}} .
$$

Proof. Fix an inner product $\langle$,$\rangle on V$. This induces an inner product on $V^{\otimes k}$, which is also denoted by $\langle$,$\rangle . Let e_{1}, \ldots, e_{d}$ be an orthonormal basis for $V$. Write $\mathcal{F}$ for the set of maps from $\{1, \ldots, k\}$ to $\{1, \ldots, d\}, \mathcal{F}_{S}$ for the restrictions to a subset $S$ of $\{1, \ldots, k\}, e_{\varphi}:=\bigotimes_{i=1}^{k} e_{\varphi(i)}$. Then $\left\{e_{\varphi}: \varphi \in \mathcal{F}\right\}$ is an orthonormal basis of $V^{\otimes k}$. Write

$$
s=\prod_{j=1}^{r} \prod_{i=1}^{a_{j}} \zeta_{i}^{j},
$$


where $\left\{\zeta_{i}^{j}: i=1, \ldots, a_{j}\right\}$ are the cycles of length $j$ in $s$. Furthermore, for any $t \in \mathfrak{S}_{k}$, write $[t]:=\{\nu=1, \ldots, k: \nu t \neq \nu\}$. Then

$$
\begin{aligned}
& \operatorname{Tr}\left(B^{\otimes k} \sigma_{k}(s)\right)=\sum_{\varphi \in \mathcal{F}}\left\langle B^{\otimes k} \sigma_{k}(s) e_{\varphi}, e_{\varphi}\right\rangle=\sum_{\varphi \in \mathcal{F}} \prod_{j=1}^{k}\left\langle B e_{j s^{-1}}, e_{j \varphi}\right\rangle \\
= & \sum_{\varphi \in \mathcal{F}} \prod_{j=1}^{r} \prod_{i=1}^{a_{j}} \prod_{l \in\left[\zeta_{i}^{j}\right]}\left\langle B e_{l\left(\zeta_{i}^{j}\right)^{-1} \varphi}, e_{l \varphi}\right\rangle=\prod_{j=1}^{r} \prod_{i=1}^{a_{j}} \sum_{\varphi \in \mathcal{F}_{\left[\zeta_{i}^{j}\right]}} \prod_{l \in\left[\zeta_{i}^{j}\right]}\left\langle B e_{l\left(\zeta_{i}^{j}\right)^{-1} \varphi}, e_{l \varphi}\right\rangle \\
= & \prod_{j=1}^{r} \prod_{i=1}^{a_{j}} \operatorname{Tr}\left(B^{j}\right)=\prod_{j=1}^{r} \operatorname{Tr}\left(B^{j}\right)^{a_{j} .}
\end{aligned}
$$

\section{Phase function for the matrix integral}

We start by rewriting the integral (5) using Weyl's integration formula, assuming for simplicity that $f$ is a class function:

$$
I_{N}(f, \alpha)=\frac{1}{|W|} \int_{T} \prod_{j=1}^{r} \operatorname{Tr}\left(\rho_{\lambda}\left(t^{j}\right)\right)^{N \alpha_{j}} f(t)|\Delta(t)|^{2} d t,
$$

where $T \subset G$ is a maximal torus, $d t$ is Haar measure on $T$, normalized as a probability measure, and $\Delta(t)$ is the Weyl denominator. We define the following function on the complexified Lie algebra $\mathfrak{t}^{\mathbb{C}}$ :

$$
k(w)=\sum_{\mu \in M_{\lambda}} m_{\lambda}(\mu) e^{2 \pi\langle\mu, w\rangle},
$$

where $w=\tau+\sqrt{-1} \varphi \in \mathfrak{t} \oplus \sqrt{-1} \mathfrak{t}=\mathfrak{t}^{\mathbb{C}}$, and linear forms in $\mathfrak{t}^{*}$ are extended complex linearly to $\mathfrak{t}^{\mathbb{C}}$. Note that the restriction of $k$ to $\sqrt{-1}$ is essentially the character of $V_{\lambda}$.

Let $d \varphi$ denote Lebesgue measure on $\mathfrak{t}$, normalized so that the fundamental domain $T_{o}$ of the integral lattice $I$ has volume 1 . Then the integral $I_{N}$ can be written in the form:

$$
I_{N}=\frac{1}{|W|} \int_{T_{o}} F(\sqrt{-1} \varphi)^{N} f(\varphi)|\Delta(\varphi)|^{2} d \varphi, \quad F(\sqrt{-1} \varphi)=\prod_{j=1}^{r} k(\sqrt{-1} j \varphi)^{\alpha_{j}}
$$

where the class function $f$ is, through the exponential map, regarded as a function on $\mathrm{t}$.

Lemma 3.1. We have the inequality

$$
|F(\sqrt{-1} \varphi)| \leq k(0)^{|\alpha|}=\left(\operatorname{dim} V_{\lambda}\right)^{|\alpha|}
$$

Equality holds if and only if $\varphi$ is in the dual lattice $\Lambda$ of the root lattice $\Lambda^{*}$.

Proof. The proof is similar to the proof of Lemma 1.4 in [TZ]. Note that the assumption about the gcd is used here.

By Lemma 3.1, the integral (13) or (15) is localized on $\operatorname{ker} \pi=\Pi(G)=\Lambda / I$. Let $g$ be a smooth cut-off function on $T$ around the unit such that the support of $g$ does not contain any element in ker $\pi$ other than the unit. Then, the translate 
$g_{h}(t)=g\left(h^{-1} t\right)$ is a cut-off function around $h \in \operatorname{ker} \pi$. Let $\varphi \in \Lambda$. Then it is easy to see that

$$
k(\sqrt{-1} j \varphi)=e^{2 \pi \sqrt{-1} j\langle\lambda, \varphi\rangle} k(0),
$$

which is not zero. Thus, around each $\varphi \in \Lambda$, we can take a branch of the logarithm to define the following function $\Phi$ :

$$
\Phi(w):=\sum_{j=1}^{r} \alpha_{j} \log k(j w), \quad w=\tau+\sqrt{-1} \varphi \in \mathfrak{t}^{\mathbb{C}},
$$

where $\varphi$ varies in a neighborhood of a point in $\Lambda$. Then, by Lemma 3.1, we can write the integral $I_{N}$ as follows:

$$
I_{N}=\frac{1}{|W|} e^{N \Phi(0)} \sum_{h \in \operatorname{ker} \pi} \int_{\mathfrak{t}} e^{N(\Phi(\sqrt{-1} \varphi)-\Phi(0))} g_{h}(\varphi) f(\varphi)|\Delta(\varphi)|^{2} d \varphi
$$

plus a term of order $O\left(e^{-c N}\right)$ for some $c>0$. To compute each of the integrals in the sum in (19), we note that

$$
\Delta\left(\varphi+\psi_{h}\right)=e^{2 \pi \sqrt{-1}\left\langle\rho, \psi_{h}\right\rangle} \Delta(\varphi)
$$

for each $h \in \operatorname{ker} \pi \cong \Lambda / I$, where $\psi_{h} \in \Lambda$ satisfies $\exp \left(\psi_{h}\right)=h$ and $\rho$ is half the sum of the positive roots. Furthermore, by (17), we have

$$
\Phi\left(\sqrt{-1}\left(\varphi+\psi_{h}\right)\right)=\Phi(\sqrt{-1} \varphi)+2 \pi \sqrt{-1} k_{\alpha}\left\langle\lambda, \psi_{h}\right\rangle .
$$

Therefore, we obtain:

Lemma 3.2. We have

$$
I_{N}=\frac{1}{|W|} \sum_{h \in \operatorname{ker} \pi} \nu_{N k_{\alpha} \lambda}(h) \int e^{N \Phi(\sqrt{-1} \varphi)} g(\varphi) f_{h}(\varphi)|\Delta(\varphi)|^{2} d \varphi
$$

plus a term of order $O\left(e^{-c N}\right)$, where $g(\varphi)$ is a cut-off function around $\varphi=0$, and $f_{h}(\varphi)=f\left(\varphi+\psi_{h}\right)$ with a representative $\psi_{h} \in \Lambda$ for $h \in \operatorname{ker} \pi$.

Next, we compute the first and second derivatives of the phase function $\Phi$ at points in $\operatorname{ker} \pi$.

Lemma 3.3. Any $\varphi \in \Lambda$ is a critical point of $\Phi$. Furthermore, the negative of the Hessian of $\Phi, H(\varphi): \mathfrak{t} \rightarrow \mathfrak{t}^{*}$, is given by $H(\varphi)=(2 \pi)^{2} l_{\alpha} A_{\lambda}$, which is independent of $\varphi \in \Lambda$ and is positive definite, where $l_{\alpha}$ and $A_{\lambda}$ are defined in (4) and (8), respectively.

Proof. That each $\varphi \in \Lambda$ is a critical point of $\Phi$ is proven by the fact that

$$
\sum_{\mu \in M_{\lambda}} m_{\lambda}(\mu) \mu=0
$$

because the left-hand side of the above is a $W$-invariant vector and $G$ is assumed to be semisimple. The fact that the linear map $A_{\lambda}: \mathfrak{t} \rightarrow \mathfrak{t}^{*}$ is positive definite is proven in [TZ].

Therefore, what we need to do is to find asymptotics of each of the integrals in (20), each of which is an integral of functions supported around the origin. However, there is a difficulty: for each integral, the origin is the unique critical point for the complex phase function $\Phi$, but the Weyl denominator $\Delta$ vanishes at the origin. In the next section, we will use ideas of Biane $([\mathrm{B}])$ and Klyachko-Kurtaran $([\mathrm{KK}])$ to circumvent this problem. 


\section{The method of Biane and KlyachKo-Kurtaran}

In this section we study the following integral, which is a slight generalization of the integral on the right-hand side of (20):

$$
J_{N}:=\int_{T} e^{N \Phi(t)} g(t)|\Delta(t)|^{2} d t
$$

where $d t$ is normalized Haar measure on the maximal torus $T$ and $\Delta$ denotes the Weyl denominator. $g$ is a compactly supported smooth function that does not vanish in the unit element of $T$. We fix a $W$-invariant inner product on $\mathfrak{t}$ such that the volume of the parallelotope determined by an orthonormal basis equals 1 . We assume that the complex-valued smooth phase function $\Phi$, which we view as a function on the complexified Lie algebra $\mathfrak{t}^{\mathbb{C}}$, satisfies the following conditions:

(i) $\operatorname{Re}(\Phi(\sqrt{-1} \varphi)) \leq \Phi(0)$, with equality if and only if $\varphi=0$;

(ii) the origin is a critical point of $\Phi$;

(iii) $H=-\partial^{2} \Phi(0): \mathfrak{t} \rightarrow \mathfrak{t}^{*}$, the negative of the Hessian of $\Phi$ at the origin, is positive definite;

(iv) in the Taylor expansion

$$
\Phi(\sqrt{-1} \varphi)-\Phi(0)=-\langle H \varphi, \varphi\rangle / 2-\sqrt{-1} \Theta(\varphi)+R_{4}(\varphi)
$$

up to fourth order, $\Theta$ is a real-valued homogeneous polynomial of degree 3 ;

(v) the linear map $H$ commutes with the action of the Weyl group $W$.

Note that the phase functions of the integrals $I_{N}$ and $K_{N}$ (see Sections 3 and [5) satisfy these conditions. We are now in a position to state the main result of this section:

Theorem 4.1. Under the above conditions on $\Phi$ and $g$ one has

$$
J_{N}=\left(\frac{2 \pi}{N}\right)^{(\operatorname{dim} G) / 2} \frac{(2 \pi)^{d} g(0) e^{N \Phi(0)}|W|}{\sqrt{\operatorname{det} H}} \kappa\left(H^{-1} \rho\right)\left(1+O\left(N^{-1 / 2}\right)\right),
$$

where $|W|$ is the order of the Weyl group $W, d$ is the number of positive roots, $\rho$ is half the sum of the positive roots, and the polynomial $\kappa$ on $\mathfrak{t}$ is defined in (7).

Proof. First of all, we normalize Lebesgue measure on $\mathfrak{t}$ so that the volume of the fundamental domain $T_{o}$ of the lattice $I$ equals 1 , and write

$$
J_{N}=e^{N \Phi(0)} \int_{T_{o}} e^{N[\Phi(\sqrt{-1} \varphi)-\Phi(0)]} g(\varphi)|\Delta(\varphi)|^{2} d \varphi .
$$

We may regard $g$ as a function on $\mathfrak{t}$ with arbitrarily small compact support around the origin, since by (i) the integrand in (24) is bounded by $e^{-c N}$ (with a constant $c>0$ ) outside a compact neighborhood of the origin. (i) and (iv) imply that for fixed $a>0$ one can choose $b>0$ such that $\operatorname{Re} \Phi(\sqrt{-1} \varphi)-\Phi(0) \leq-b\langle H \varphi, \varphi\rangle$ for $|\varphi| \leq a$. Substituting (22) into $J_{N}$ and changing the variable $\varphi$ to $N^{-1 / 2} \varphi$, we have

$$
\begin{aligned}
J_{N} & =N^{-(\mathrm{rk} G) / 2} e^{N \Phi(0)} \\
& \times \int e^{-\langle H \varphi, \varphi\rangle / 2-\sqrt{-1} N \Theta\left(N^{-1 / 2} \varphi\right)+N R_{4}\left(N^{-1 / 2} \varphi\right)} g\left(N^{-1 / 2} \varphi\right)\left|\Delta\left(N^{-1 / 2} \varphi\right)\right|^{2} d \varphi .
\end{aligned}
$$

As in [B], TZ], using the identity $\Delta=\prod_{\alpha \in \Phi_{+}}\left(e^{\pi \sqrt{-1} \alpha}-e^{-\pi \sqrt{-1} \alpha}\right)$, it is easy to see that

$$
\Delta\left(N^{-1 / 2} \varphi\right)=(2 \pi \sqrt{-1})^{d} N^{-d / 2} \kappa(\varphi)\left(1+O\left(N^{-1}|\varphi|^{2}\right)\right)
$$


We note that $g\left(N^{-1 / 2} \varphi\right)=g(0)\left(1+O\left(N^{-1 / 2}|\varphi|\right)\right)$. Substituting these formulas into (25) and introducing a smooth cut-off function $\chi$ such that $\chi=1$ around the support of $g$, we obtain

$$
\begin{aligned}
& J_{N}=\frac{(2 \pi)^{2 d} g(0) e^{N \Phi(0)}}{N^{d+(\operatorname{rk} G) / 2}} \\
& \times \int e^{-\langle H \varphi, \varphi\rangle / 2-\sqrt{-1} N^{-1 / 2} \Theta(\varphi)+N R_{4}\left(N^{-1 / 2} \varphi\right)} \chi\left(N^{-1 / 2} \varphi\right)|\kappa(\varphi)|^{2} d \varphi\left(1+O\left(N^{-1 / 2}\right)\right) .
\end{aligned}
$$

Here, we note that $g$ and its derivatives are bounded on $\mathfrak{t}$, and that the exponential in the integrand is bounded by $e^{-b\langle H \varphi, \varphi\rangle}$ if $|\varphi| / N^{1 / 2} \leq a$. As indicated above, we may assume that $g\left(N^{-1 / 2} \varphi\right)=0$ for $|\varphi| / N^{1 / 2} \geq a$.

Next, as in [TZ, we divide the integral in (26) into several parts as follows. We set $E_{N}(\varphi):=e^{-\langle H \varphi, \varphi\rangle / 2-\sqrt{-1} N^{-1 / 2} \Theta(\varphi)}$ and write

$$
J_{N}=\frac{(2 \pi)^{2 d} g(0) e^{N \Phi(0)}}{N^{d+(\operatorname{rk} G) / 2}}\left(\sum_{j=1}^{4} I_{j}(N)\right)\left(1+O\left(N^{-1 / 2}\right)\right)
$$

where the integrals $I_{j}(N), j=1,2,3,4$ are given by

$$
\begin{gathered}
I_{1}(N)=\int E_{N}(\varphi)|\kappa(\varphi)|^{2} d \varphi \\
I_{2}(N)=\int E_{N}(\varphi)\left(e^{N R_{4}\left(N^{-1 / 2} \varphi\right)}-1\right) \chi\left(N^{-1 / 4} \varphi\right)|\kappa(\varphi)|^{2} d \varphi \\
I_{3}(N)=\int E_{N}(\varphi) e^{N R_{4}\left(N^{-1 / 2} \varphi\right)}\left(1-\chi\left(N^{-1 / 4} \varphi\right)\right) \chi\left(N^{-1 / 2} \varphi\right)|\kappa(\varphi)|^{2} d \varphi, \\
I_{4}(N)=\int E_{N}(\varphi)\left(\chi\left(N^{-1 / 4} \varphi\right)-1\right)|\kappa(\varphi)|^{2} d \varphi
\end{gathered}
$$

where we used the relation $\chi\left(N^{-1 / 4} \varphi\right) \chi\left(N^{-1 / 2} \varphi\right)=\chi\left(N^{-1 / 4} \varphi\right)$ for sufficiently large $N$.

Now, the integrand in $I_{2}(N)$ vanishes for $|\varphi| \geq c N^{1 / 4}$, and thus we have $e^{N R_{4}\left(N^{-1 / 2} \varphi\right)}=O(1)$ and $N R_{4}\left(N^{-1 / 2} \varphi\right)=|\varphi|^{4} O(1 / N)$. Hence, $\left(e^{N R_{4}\left(N^{-1 / 2} \varphi\right)}-1\right)$ is bounded by $c|\varphi|^{4} O\left(\frac{1}{N}\right)$. But we have $\left|E_{N}(\varphi)\right|=e^{-\langle H \varphi, \varphi\rangle / 2}$, and hence $|\varphi|^{4} E_{N}(\varphi)$ is integrable uniformly in $N$. Thus we have $I_{2}(N)=O(1 / N)$. As to $I_{3}(N)$, the function $E_{N}(\varphi) e^{N R_{4}\left(N^{-1 / 2} \varphi\right)}$ is dominated by $e^{-b\langle H \varphi, \varphi\rangle}$ wherever the integrand does not vanish. Since $\chi\left(N^{-1 / 4} \varphi\right)=1$ for $|\varphi| \leq c N^{1 / 4}$ for some $c>0$, we easily have $I_{3}(N)=O\left(N^{c_{1}} e^{-c_{2} N^{1 / 2}}\right)$ for some $c_{1}, c_{2}>0$. Similarly, we have $I_{4}(N)=O\left(N^{c_{1}} e^{-c_{2} N^{1 / 2}}\right)$.

Finally, we consider the integral $I_{1}(N)$. Note that $e^{-\sqrt{-1} \Theta(\varphi) N^{-1 / 2}}=1+$ $O\left(|\varphi|^{3} / N^{1 / 2}\right)$ since $\Theta(\varphi)$ is real. Thus, invoking the identity

$$
\int_{\mathfrak{t}} e^{-\langle H x, x\rangle / 2}|\kappa(x)|^{2} d x=\frac{(2 \pi)^{(\mathrm{rk} G) / 2}|W| \kappa\left(H^{-1} \rho\right)}{\sqrt{\operatorname{det} H}},
$$


known as Mehta's conjecture and proven in $[\mathrm{Mc}$ and $\mathrm{Op}$, we have

$$
\begin{aligned}
I_{1}(N) & =\int e^{-\langle H \varphi, \varphi\rangle}|\kappa(\varphi)|^{2} d \varphi\left(1+O\left(N^{-1 / 2}\right)\right) \\
& =\frac{(2 \pi)^{(\operatorname{rk} G) / 2}|W| \kappa\left(H^{-1} \rho\right)}{\sqrt{\operatorname{det} H}}\left(1+O\left(N^{-1 / 2}\right)\right),
\end{aligned}
$$

which completes the proof.

\section{Proof of the main Results}

Theorem 1 is a direct application of Theorem 4.1 with Lemma 3.3 to the integral on the right-hand side of (20). To prove Theorem 2, we proceed as in Section 3 and use the Weyl integration formula to obtain

$$
K_{N}=\frac{1}{|W|} \int_{T} J(t)^{N} f(t)|\Delta(t)|^{2} d t, \quad J(t)=\prod_{j=1}^{r} \operatorname{Tr}\left(\rho_{\lambda}\left(t^{j}\right)\right)^{\alpha_{j}}{\overline{\operatorname{Tr}\left(\rho_{\lambda}\left(t^{j}\right)\right.}}^{\beta_{j}} .
$$

We also apply Theorem 4.1 to find asymptotics of the integral $K_{N}$ as follows. As in Lemma 3.1, we have

$$
|J(\sqrt{-1} \varphi)| \leq k(0)^{|\alpha|+|\beta|}=\left(\operatorname{dim} V_{\lambda}\right)^{|\alpha|+|\beta|},
$$

with equality if and only if $\varphi \in \Lambda$. We define

$$
\Psi(w)=\sum_{j=1}^{s}\left[\alpha_{j} \log k(j w)+\beta_{j} \log k(j \bar{w})\right], \quad w=\tau+\sqrt{-1} \varphi \in \mathfrak{t}^{\mathbb{C}},
$$

around each $\varphi \in \Lambda$. Next, we need to compute the Hessian of $\Psi$ at $\varphi \in \Lambda$. We note that, for $\varphi \in \Lambda$, we have

$$
\begin{gathered}
k(\sqrt{-1} j \varphi)=e^{2 \pi \sqrt{-1} j\langle\lambda, \varphi\rangle} k(0), \quad(\partial k)(\sqrt{-1} j \varphi)=0, \\
\partial^{2}(\log k(\sqrt{-1} j \varphi))=-4 \pi^{2} j^{2} A_{\lambda} .
\end{gathered}
$$

Therefore, we have the following lemma:

Lemma 5.1. Each $\varphi \in \Lambda$ is a critical point of $\Psi$. The negative of the Hessian of $\Psi$, denoted $D(\varphi)$, is given by $D(\varphi)=(2 \pi)^{2}\left(l_{\alpha}+l_{\beta}\right) A_{\lambda}$, and hence $D=D(\varphi)$ does not depend on $\varphi \in \Lambda$, and is positive definite.

This time, the term involving $2 \pi \sqrt{-1} j\left\langle\lambda, \psi_{h}\right\rangle$ disappears because of the assumption $\sum j \alpha_{j}=\sum j \beta_{j}$. We thus have

$$
K_{N}=\frac{1}{|W|} \sum_{h \in \operatorname{ker} \pi} \int_{\mathfrak{t}} e^{N \Psi(\sqrt{-1} \varphi)} g(\varphi) f_{h}(\varphi)|\Delta(\varphi)|^{2} d \varphi,
$$

where $f_{h}$ is defined in Lemma 3.2 . The assumption of Theorem 4.1 is satisfied by the phase function $\Psi$, and applying it, we obtain Theorem 2 ,

\section{ACKNOWLEDGEMENTS}

Both authors have been supported by DFG via SFB/TR 12. The first author acknowledges support of JSPS in the framework of the Japanese-German programme on infinite-dimensional harmonic analysis. The second author has been supported by JSPS Grant-in-Aid for Scientific Research no. 18740089. This article was written during his stay at Ruhr-Universität Bochum. He would like to thank the people in the mathematics department, notably Prof. Alan Huckleberry, for their hospitality. 


\section{REFERENCES}

[BR] J. Baik and E. Rains, Algebraic aspects of increasing subsequences, Duke Math. J. 109 (2001), 1-65. MR1844203 (2002i:05119)

[B] P. Biane, Estimation asymptotique des multiplicités dans les puissances tensorielles d'un g-module, C. R. Acad. Sci. Paris Sér. I Math. 316 (8) (1993), 849-852. MR.1218274 (94a:17004)

[CS] B. Collins and P. Śniady, Representations of Lie groups and random matrices, math.PR/ 0610285.

[DE] P. Diaconis and S. N. Evans, Linear functionals of eigenvalues of random matrices, Trans. Amer. Math. Soc. 353 (2001), no. 7, 2615-2633. MR1828463(2002d:60003)

[DS] P. Diaconis and M. Shahshahani, On the eigenvalues of random matrices, J. Appl. Probab. 31A (1994), 49-62. MR1274717 (95m:60011)

[K] G. Kuperberg, Random words, quantum statistics, central limits, random matrices, Methods Appl. Anal. 9 (2002), 99-118. MR1948465 (2003k:60020)

[KK] A. Klyachko and E. Kurtaran, Some identities and asymptotics for characters of the symmetric group, J. Algebra 206 (1998), 413-437. MR1637064 (99h:14033)

[Mc] I. G. Macdonald, Some conjectures for root systems, SIAM J. Math. Anal. 13 (1982), 9881007. MR674768 (84h:17006a)

[Op] E. M. Opdam, Some applications of hypergeometric shift operators, Invent. Math. 98 (1989), 1-18. MR:1010152 (91h:33024)

[Ra] A. Ram, Characters of Brauer's centralizer algebras, Pacific J. Math. 169 (1995), 173-200. MR $1346252(96 \mathrm{k}: 20020)$

[St] M. Stolz, On the Diaconis-Shahshahani method in random matrix theory, J. Algebraic Combin. 22 (2005), 471-491. MR.2191648

[TZ] T. Tate and S. Zelditch, Lattice path combinatorics and asymptotics of multiplicities of weights in tensor powers, J. Funct. Anal. 217 (2004), 402-447. MR2102573 (2005h:22023)

[Wy] H. Weyl, The Classical Groups. Their Invariants and Representations, Princeton University Press, Princeton, NJ, 1953, repr. 1997. MR.1488158 (98k:01049)

Fakultät für Mathematik, Ruhr-Universität Bochum, NA 4/32, D-44780 Bochum, GERMANY

E-mail address: michael.stolz@ruhr-uni-bochum.de

Graduate School of Mathematics, Nagoya University, Furo-cho, Chikusa-ku, Nagoya, 464-8602 JAPAN

E-mail address: tate@math.nagoya-u.ac.jp 